folding the theoretical distribution of Drechsel and Maximon ${ }^{6}$ and of Liou and $\mathrm{Cho}^{8}$ with the experimental noncoplanar resolution. The Drechsel-Maximon prediction ${ }^{6}$ was based on the Hamada-Johnston potential; an almost identical result was obtained using the Reid potential. The Liou-Cho calculation ${ }^{8}$ was also based on the Hamada-Johnston potential, but a relativistic spin correction was included. The agreement with either of these calculated distributions is good. This agreement validates the use of the Drechsel-Maximon distribution for correcting experimental data for noncoplanarity in this energy region. ${ }^{5,11}$

Figure 3 shows the energy distribution of events plotted without regard to angle of noncoplanarity. A total of $370 \pm 26$ events is attributed to PPB. The Drechsel-Maximon noncoplanar distribution was used to calculate a multiplicative factor of 1.384 to correct for the effect of noncoplanar acceptance. This leads to a coplanar cross section

$$
\frac{d^{2} \sigma_{\mathrm{exp}}}{d \Omega_{L} d \Omega_{R}}=2.32 \pm 0.20 \mu \mathrm{b} / \mathrm{sr}^{2}
$$

If the correction factor is based on the LiouCho distribution, the coplanar cross section is $3.3 \%$ lower. Our result confirms earlier Oak Ridge measurements, which are generally below currently accepted predictions.

Table I shows our coplanar cross section in comparison with theoretical $30^{\circ}-30^{\circ}$ cross sections estimated for $64.4 \mathrm{MeV}$ from calculations at 61.7 and $65 \mathrm{MeV}$. The discrepancy between this measurement and all these theoretical calculations is outside the experimental error.

$\dagger$ Research sponsored by the U. S. Atomic Energy Commission under contract with Union Carbide Corp.

*Oak Ridge Graduate Fellow from Tulane University, under appointment from Oak Ridge Associated Universities, 1966-1968.

${ }^{1}$ E. Nyman, Phys. Rev. 170, 1628 (1968).

${ }^{2}$ M. D. Miller, M. S. Sher, P. Signell, N. R. Yoder and D. Marker, Phys. Lett. 30B, 157 (1969).

${ }^{3}$ R. E. Warner, Can. J. Phys. 44, 1225 (1966).

${ }^{4}$ I. Slaus, J. W. Verba, J. R. Richardson, R. F. Carlson, W. T. H. van Oers, and L. S. August, Phys. Rev. Lett. 17, 536 (1966).

${ }^{5}$ D. L. Mason, M. L. Halbert, and L. C. Northcliffe, Phys. Rev. 176, 1159 (1968).

${ }^{6}$ D. Drechsel and L. C. Maximon, Ann. Phys. (New York) 49,403 (1968).

${ }^{7}$ R. Baier, H. Kühnelt, and P. Urban, Nucl. Phys. B 11, 675 (1969).

${ }^{8}$ M. K. Liou and K. S. Cho, unpublished.

${ }^{9}$ B. Gottschalk, W. J. Shlaer, and K. H. Wang, Nuc1. Phys. A 94, 491 (1967).

${ }^{10}$ M. L. Halbert, D. L. Mason, and L. C. Northcliffe, Phys. Rev. 168, 1130 (1968).

${ }^{11}$ D. Drechsel, L. C. Maximon, and R. E. Warner, Phys. Rev. 181, 1720 (1969).

${ }^{12}$ V. R. Brown, Phys. Rev. 177, 1498 (1969), and private communication.

${ }^{13}$ D. Marker and P. Signell, Phys. Rev. 185, 1286 (1969).

${ }^{14} \mathrm{~J}$. H. McGuire, A. H. Cromer, and M. I. Sobel, Phys. Rev. 179, 948 (1969).

${ }^{15} \mathrm{G}$. Schiffrer and D. Drechsel, unpublished.

${ }^{16}$ R. A. Bryan and B. Scott, Phys. Rev. 177, 1435 (1969).

\title{
Borel Summability of the Ground-State Energy in Spatially Cutoff $\left(\varphi^{4}\right)_{2}^{\dagger}$
}

\author{
Barry Simon \\ Jadwin Physical Laboratory, Princeton University, Princeton, New Jersey 08540
}

(Received 1 October (1970)

\begin{abstract}
We show that the ground-state energy for a Hamiltonian $H_{0}+\int g(x): \varphi^{4}(x): d x\left(g \in L^{1} \cap L^{2}\right.$; $g \geqslant 0 ; H_{0}=$ free Hamiltonian for Bose particle of mass $m$ in space-time of two dimensions) may be determined from the Feynman perturbation series by the method of Borel summability. This demonstrates that summability methods can be applicable to divergent series in systems with a continuous infinity of degrees of freedom.
\end{abstract}

We prove here that for a spatially cutoff $\left(\varphi^{4}\right)_{2}$ theory ${ }^{1}$ the ground-state energy can be recovered from the (Feynman) perturbation series by the method of Borel summability. This represents a merging of two trends in mathematical physics. In the first place, considerable effort has gone into the study of the analytic properties of the levels of the anharmonic oscillator ${ }^{2}$ and the proof that in various cases, one can recover the levels from the perturbation series by the $\mathrm{Pade}^{3}$ or $\mathrm{Borel}^{4.5}$ method despite the 
fact that the series is divergent. ${ }^{6}$ The second trend involves the construction of two-dimensional field theories. Using early results of Jaffe and Nelson, ${ }^{7}$ Glimm and Jaffe developed ${ }^{8}$ an impressive amount of lore for $\left(\varphi^{4}\right)_{2}$ field theories without cutoffs by studying the cutoff theories. Various refinements and extensions have been made by Cannon and Jaffe, Rosen, Segal, and Simon and Höegh-Krohn. ${ }^{9}$

We deal here with Hamiltonians $H_{0}+\beta V$ with ${ }^{10} H_{0}=\int\left(k^{2}+m^{2}\right)^{1 / 2} a^{*}(k) a(k) d k$ and ${ }^{11} V=\int g(x): \varphi^{4}(x): d x$ with $g \in L^{1} \cap L^{2} ; g \geqslant 0$. This theory has a continuous infinity of degrees of freedom and is known ${ }^{12}$ to have divergent Feynman perturbation series. Our proof also applies without change to a theory with box cutoff rather than $g(x)$ cutoff.

We establish the Borel summability by using Watson's theorem ${ }^{13}$ which says the following: Given any function $f(\beta)$, analytic in $\{\beta|\arg \beta|<\theta ; 0<|\beta|<B\}=D$ with $\theta>\pi / 2$, suppose $f(\beta)$ has an asymptotic series $f \sim \sum a_{n} \beta^{n}$ with strong control over the remainder:

$$
\left|f(\beta)-\sum_{n=0}^{N} a_{n} \beta^{n}\right|<A \sigma^{N+1}(N+1) !|\beta|^{N+1}
$$

uniform for $\beta \in D$ and $n$, where $\sigma$ and $A$ are constants. Then the asymptotic series in Borel summable to $f$, i.e., the function

$$
G(\beta)=\sum_{n=0}^{\infty} \frac{a_{n}}{(n+1) !} \beta^{n}
$$

(defined for $|\beta|<\sigma^{-1}$ ), has an analytic continuation to $\{\beta|\arg \beta|<\theta-\pi / 2\}$; and for $|\beta|<B$, $\arg \beta \mid<\theta-\pi / 2$,

$$
f(\beta)=\int_{0}^{\infty} e^{-x} G(\beta x) d x .
$$

In particular, ${ }^{14}$ we prove that the ground-state energy is completely determined by the Feynman series.

Our proof is in four steps:

(1) For any $\theta<\pi$, and any $\lambda \notin \subset \operatorname{spec}\left(H_{0}\right)$ [we denote the spectrum of an operator $A$ by $\left.\operatorname{spec}(A)\right] \underline{\left(H_{0}+\beta V\right.}$ $-\lambda)^{-1} \rightarrow\left(H_{0}-\lambda\right)^{-1}$ in norm as $|\beta| \rightarrow 0$ uniformly in $\operatorname{larg} \beta \mid<\theta$. This represents a critical improvement of the results of Simon and Höegh-Krohn ${ }^{9}$ who prove a similar result for $\left(\varphi^{2 m}\right)_{2}$ theories if $\theta<\pi / 2$. By standard operator theory, ${ }^{15}$ it suffices to prove this for some fixed $\lambda$. By realizing $V$ as a multiplication operator, ${ }^{16}$ we can write $V=V_{+}-V_{-}>0 ; V_{+} V_{-}=0$. Let $|V|=V_{+}+V_{-}, U=|V| / V$, and $W=|V|^{1 / 2}$. Since

$$
\left\|\left(H_{0}+V-\lambda\right)^{-1}-\left(H_{0}-\lambda\right)^{-1}\right\|=|\beta|\left\|\left(H_{0}-\lambda\right)^{-1} W U W\left(H_{0}+\beta V-\lambda\right)^{-1}\right\|,
$$

it is sufficient to prove that $\left\|\left(H_{0}-\lambda\right)^{-1} W\right\|$ and $|\beta|^{1 / 2}\left\|W\left(H_{0}+\beta V-\lambda\right)^{-1}\right\|$ are bounded uniformly for some $\lambda$ and for all $\beta$ in the sector of interest. Since ${ }^{17} W^{2}=|V| \leqslant a N^{2}+b \leqslant a H_{0}{ }^{2}+b$ for some $a$ and $b,\left(H_{0}-\lambda\right)^{-1} W^{2}$ $\times\left(H_{0}-\bar{\lambda}\right)^{-1} \leqslant C_{\lambda}^{2}$ for any $\lambda \notin \operatorname{spec}\left(H_{0}\right)$ so $\left\|\left(H_{0}-\lambda\right)^{-1} W\right\|<C_{\lambda}$. To prove the other bound, we use sectorial considerations. Pick $B$ so that for all $\gamma$ with $|\gamma|=|\beta| B$, and all $\beta$ with $|\arg \beta|<\theta, \arg (\beta+\gamma)<(\pi+\theta) / 2$.

Consider $H_{0}+\beta V+\gamma W^{2}=H_{0}+(\gamma+\beta) V_{+}+(\beta-\gamma) V_{-}$. By arguments of Simon and Höegh-Krohn, ${ }^{9}$ this is uniformly sectorial for all $\beta$ with $\arg \beta|<0,| \beta \mid<1$, all $\gamma$ with $|\gamma|=|\beta| B$; that is, there is an $\alpha$ with $|\alpha|$ $=1$ and a $b$ so that for all vectors $u$ and all $\operatorname{such} \beta, \gamma$,

$$
\operatorname{Re}\left[\alpha\left\langle u,\left(H_{0}+\beta V+\gamma W^{2}\right) u\right\rangle+b\langle u, u\rangle\right]>0,
$$

or

$$
|\beta| B\left|\left\langle u, W^{2} u\right\rangle\right|<\operatorname{Re}\left[\alpha\left\langle u,\left(H_{0}+\beta V-\lambda\right) u\right\rangle\right]+c\langle u, u\rangle .
$$

Since the $H_{0}+\beta V$ are known to be uniformly sectorial, we can find $\lambda \not \subset \operatorname{spec}\left(H_{0}+\beta V\right)$, all relevant $\beta$, with $\left(H_{0}+\beta V-\lambda\right)^{-1}$ uniformly bounded. Then

$$
\begin{aligned}
|\beta| B\left\|W\left(H_{0}+\beta V-\lambda\right)^{-1} u\right\|^{2} & \leqslant \operatorname{Re}\left[\alpha\left\langle\left(H_{0}+\beta V-\lambda\right)^{-1} u, u\right\rangle\right]+b\left\|\left(H_{0}+\beta V-\lambda\right)^{-1} u\right\|^{2} \\
& \leqslant\|u\|^{2}\left[\left\|\left(H_{0}+\beta V-\lambda\right)^{-1}\right\|+b\left\|\left(H_{0}+\beta V-\lambda\right)^{-1}\right\|^{2}\right] .
\end{aligned}
$$

This proves the uniform bound on $|\beta|^{1 / 2}\left|W\left(H_{0}+\beta V-\lambda\right)^{-1}\right|$ and hence completes the proof of step 1 .

(2) For any $\theta<\pi$, there is a $B$ so that $H+\beta V$ has only one eigenvalue near 0 for $|\beta|<B, \quad|\arg \beta|<\theta$; and the energy of this eigenvalue is analytic in $\{\beta|\arg \beta|<\theta,|\beta|<B\}$. This is a direct consequence ${ }^{15}$ of (1) and the fact that $H_{0}$ has 0 as an isolated, nondegenerate eigenvalue. 
(3) The Feynman series for the ground-state energy is asymptotic as $|\beta| \rightarrow 0$ uniformly in $\arg \beta \mid<\theta$, any $\theta<\pi$. Once one has the control of the resolvent given by (1), this follows as in the work of Simon and Höegh -Krohn. ${ }^{9}$

(4) One has strong control over the remainder to the asymptotic series, i.e., if $E(\beta)$ is the groundstate energy and $a_{n}$ the Rayleigh-Schrödinger (=Feynman!) coefficients, then, given $\theta<\pi$, there are $A, \sigma, B$ so that

$$
\left|E(\beta)-\sum_{n=0}^{N} a_{n} \beta^{n}\right|<A \sigma^{n+1}(N+1) !|\beta|^{n+1}
$$

for all $\beta$ with $|\beta|<B, \arg \beta \mid<\theta$. By arguments identical to those used by Graffi, Grecchi, and Simon, ${ }^{4}$ it is enough to prove

$$
\left\|\left[V\left(H_{0}-\lambda\right)^{-1}\right]^{n+1} \Omega_{0}\right\|<A \sigma^{n+1}(n+1) !
$$

for some $A$ and $\sigma$ and all $n$, all $\lambda$ with $|\lambda|=\frac{1}{2} m$ [that is, once (1) is proven]. Write

$$
V=\sum_{j=0}^{4} V_{j}
$$

where each $V_{j}$ has $j$ creation operators and $4-j$ annhilation operators. Write $\left\|\left[V\left(H_{0}-\lambda\right)^{-1 n+1}\right] \Omega_{0}\right\|$ as $5^{n+1}$ terms of the form

$$
\begin{aligned}
\left\|V_{j_{1}}\left(H_{0}-\lambda\right)^{-1} V_{j_{2}}\left(H_{0}-\lambda\right)^{-1} \cdots V_{j_{n+1}}\left(H_{0}-\lambda\right)^{-1} \Omega_{0}\right\| & =\left\|\prod_{k=1}^{n+1}(N+1)\left[(N+1)^{-1} V_{j_{k}}(N+1)^{-1}\right]\left[(N+1)\left(H_{0}-\lambda\right)^{-1}\right] \Omega_{0}\right\| \\
& \leqslant \prod_{k=1}(4 k+1)\left\|\left[(N+1)^{-1} V_{j_{k}}(N+1)^{-1}\right](N+1)\left(H_{0}-\lambda\right)^{-1} \Omega_{0}\right\| \\
& \leqslant 5^{n+1}(n+1) ! \sigma^{n+1}
\end{aligned}
$$

with $^{18}$

$$
\sigma=\left[\max _{j=0, \cdots, 4}\left|(N+1)^{-1} V_{j}(N+1)^{-1}\right|\right] \sup _{|\lambda|=\frac{1}{2} n}\left|(N+1)\left(H_{0}-\lambda\right)^{-1}\right| .
$$

This completes the proof of (4) and thereby by Watson's theorem of the Borel summability.

$\dagger$ Research partially sponsored by the U. S. Air Force Office of Scientific Research under Contract No. AF 49(638) 1545 .

${ }^{1}$ That is, a theory with interaction Lagrangian -: $\varphi^{4}(x)$ : in space-time of two dimensions (1 space +1 time); spatially cutoff means that the formal object $\int: \varphi^{4}(x): d x$ is replaced with the self-adjoint operator $\int g(x): \varphi^{4}(x): d x$.

${ }^{2}$ J. J. Loeffel and A. Martin, to be published; B. Simon, Ann. Phys. (New York) 58 , 76 (1970); A. S. Wightman, Colloq. Int. Centre Nat. Rech. Sci. 181, 63 (1970). All these rigorous studies use intuition derived from the ap-

proximations in C. Bender and T. T. Wu, Phys. Rev. 184, 1231 (1969).

${ }^{3}$ J. J. Loeffel, A. Martin, B. Simon, and A. S. Wightman, Phys. Lett. 30B, 656 (1969).

${ }^{4}$ S. Graffi, V. Grecchi, and B. Simon, Phys. Lett. 32B, 631 (1970).

${ }^{5}$ While the Pade method is far superior for computational purposes, it is much harder to prove applicable. For finite-dimensional oscillators, the Padé method is only known (Ref.3) to work for one-dimensional $x^{4}$ and $x^{6}$ perturbations; the Borel method works for $x^{2 m}$ perturbations and in $n$ dimensions.

${ }^{6}$ See Bender and Wu, Ref. 2, or Simon, Ref. 2.

${ }^{7}$ A. Jaffe, thesis, Princeton University, 1965 (unpublished); E. Nelson, in Mathematical Theory of Elementary Particles, edited by R. Goodman and I. Segal (M. I. T. Press, Cambridge, Mass., 1966).

${ }^{8}$ J. Glimm and A. Jaffe, Phys. Rev. 176, 1945 (1968), and Commun. Pure Appl. Math. 22, 401 (1969), and Ann. Math. 91,362 (1970), and to be published.

${ }^{9} \mathrm{~J}$. Cannon and A. Jaffe, to be published; L. Rosen, Commun. Math. Phys. 16, 157 (1970), and to be published; I. Segal, Bull. Amer. Math. Soc. 75, 1390 (1969), and to be published; B. Simon and R. Hỏegh-Krohn, to be published.

${ }^{10} d k$ and $d x$ refer to one-dimensional integrations.

${ }^{11}$ The basic result depends on $\varphi^{4}$ (as opposed to $\varphi^{2 m}$ ) in two ways. First, we use inequalities $|V|<a H_{0}^{2}+b$ in step (1) below to prove $\pi-\epsilon$ analyticity; secondly, we prove an $n$ ! growth of certain coefficients. It may be possible to prove $\pi-\epsilon$ analyticity for $\varphi^{2 m}$, but for $\varphi^{2 m}$ theories the analogous coefficients almost surely have $[n(m-1)] !$ growth. To apply Borel methods to such a series one needs $\frac{1}{2} \pi(m-1)+\epsilon$ analyticity which requires some analyticity off the 
first sheet if $\boldsymbol{m}>2$.

${ }^{12}$ A. Jaffe, Commun. Math. Phys. 1, 127 (1965).

${ }^{13}$ See, e.g., G. Hardy, Divergent Series (Oxford Univ., Oxford, England, 1949).

${ }^{14}$ We regard the fact that the energy level is uniquely determined by the asymptotic series as the crucial result of this note. For both Pade and Borel summability, the required uniqueness follows from Carleman's criterion: If $|f(x)|<a_{n}|z|^{n}$ for all $z$ in a region $\left\{z|| \arg z\left|<\frac{1}{2} \pi\right| z \mid<B\right\}$ where $f$ is analytic, and if

$$
\sum_{n=1}^{\infty} a_{n}^{-1 / n=\infty}
$$

then $f=0$. Thus the unique determination from the series follows from our strong bound on the remainder.

${ }^{15} \mathrm{~T}$. Kato, Perturbation Theory for Linear Operators (Springer, Berlin, 1966).

${ }^{16}$ That $V$ can be so realized is the content of the spectral theorem; a particular such realization on " $Q$ space" is basic to some results in Refs. 8 and 9.

${ }^{17}$ That $V=V_{+}-V$. obeys $V<a^{\prime} N^{2}+b^{\prime}$ is an "elementary $N_{\tau}$ estimate" of the type employed by Glimm and Jaffe; it depends on the fact that $V$ is a sum of Wick-ordered products of four creation and annhilation operators integrated with square integral kernels. That $2 V-<a^{\prime \prime} N+b^{\prime \prime}<a^{\prime \prime} N^{2}+b^{\prime \prime}$ is a result of the type first proven by Nelson (Ref. 7) and simplified by Segal (Ref. 9).

${ }^{18}$ That $\left\|(N+1)^{-1} V_{j}(N+1)^{-1}\right\|$ is finite is the $N_{\tau}$ estimate referred to in note 17 above. Since $(N+1)\left(H_{0}-\lambda\right)^{-1}$ acts as $(n+1) / \sum_{j=1}\left[w\left(k_{i}\right)-\lambda\right]$ on the $n$-particle space with $|\lambda|=\frac{1}{2} m$ and each $w\left(k_{i}\right)>m,\left\|(N+1)\left(H_{0}-\lambda\right)^{-1}\right\|<$ some constant independent of $\arg \lambda$.

\title{
Predictions for High-Energy Elastic and Inelastic Scatterings in $\varphi^{3}$ Theory*
}

\author{
Shau-Jin Chang \\ Physics Department, University of Ilinois, $\dagger$ Urbana, Mlinois 61801, and Stanford Linear Accelerator Center, \\ Stanford University, Stanford, California 94305 \\ and \\ Tung-Mow Yan $\ddagger$ \\ Stanford Linear Accelerator Center, Stanford University, Stanford, Califormia 94305
}

(Received 10 August 1970)

\begin{abstract}
We study high-energy elastic and inelastic processes in a $\varphi^{3}$ theory based on the $s-$ channel iterations of $t$-channel ladder diagrams. The main results are the following: (a) The total cross section goes to zero, a constant, or $\sim(\ln s)^{2}$ for the coupling constant being smaller than, equal to, or larger than a critical value. (b) Inelastic differential cross sections are computed and the $s$-channel unitarity is explicitly verified. (c) Oneparticle spectrum, multiplicity, number distribution, etc. are presented. The implications of these results to hadron physics are discussed.
\end{abstract}

Because of lack of a better alternative to the conventional perturbation expansion, quantum field theory has not been proved to be useful in the analysis of high-energy behavior of elastic and inelastic scatterings in strong interactions. As the recently developed infinite-momentum technique permits one to handle the leading highenergy behavior of a very wide class of Feynman diagrams,$^{1-4}$ it may be hoped that study of certain field-theory models will at least reveal some general qualitative features concerning the questions mentioned above. Much work along this line has been done by many authors. ${ }^{3-5}$ On the basis of their results of high-order calculations of the elastic-scattering amplitude in massive quantum electrodynamics (QED), ${ }^{3}$ Cheng and $\mathrm{Wu}$ recently made a number of predictions on the elastic-scat- tering amplitude, the differential and integrated elastic cross sections, and the total cross section for hadron-hadron scattering at infinite energy. One may hope that a simple model with $\varphi^{3}$ coupling will also give the same qualitative predictions as QED; furthermore, the simplicity of the model allows one to draw more physical consequences. In this Letter we report some predictions of the simple model with a $\varphi^{3}$ coupling for elastic and inelastic hadron scattering at very high energies.

Our model is defined as follows: For the elastic-scattering amplitude we first summed the leading terms in each order of perturbation of the $t$-channel straight ladders plus those obtained by interchange of the Mandelstam variables $s$ and $u$. We then performed the $s$-channel iteration 